\title{
A RELAÇÃO UNIVERSIDADE E SERVIÇOS DE SAÚDE - CONSTRUINDO POSSIBILIDADES DE TRABALHO
}

Silvana Martins Mishima * Maria Cecília Puntel de Almeida ** Tereza Cristina Scatena Villa*

Cinira Magali Fortuna *** Maria Lúcia Rimoli Kimura*** Maria José Bistafa Ferreira***

MISHIMA, S.M. et al. A relação universidade e serviços de saúde - construindo possibilidades de trabalho. Rev.latinoam.enfermagem, Ribeirão Preto, v. 5, n. 2, p. 17-22, abril 1997.

Este ensaio visa relatar a experiência compartilhada de construção de uma relação interinstitucional Universidade $e$ serviço de saúde, apontando as possibilidades e dificuldades deste caminho.

UNITERMOS: pesquisa, pesquisa em saúde

\section{INTRODUÇÃO}

Em resposta às inúmeras inquietações no campo do ensino, assistência e pesquisa, a Universidade tem buscado maior integração com os serviços a fim de aproximar a teoria da realidade prática, esta às vezes é bem sucedida e em outras não, sendo as limitações muito grandes.

Estas limitações dizem respeito, principalmente ao fato, de que nestas organizações - a Universidade de um lado e os serviços de saúde de outro - são desenvolvidos processos de trabalho distintos.

O trabalho da academia "visa atender a uma finalidade de formação de agentes que se dirigirão ao mercado de trabalho $e$ de produção de conhecimentos através da atividade de pesquisa, que, em última instância, será 'consumida' pelos serviços de saúde. Já os profissionais de saúde constituem-se em agentes envolvidos em um processo, cuja finalidade primeira volta-se para o atendimento das necessidades de cura ou de atenção à saúde de indivíduos ou grupos, processo este constituído no limite do técnico e político” (MISHIMA, 1995, p. 104)

Há, portanto, o estabelecimento de processos de trabalho distintos para o atendimento de finalidades igualmente diferenciadas, o que parece levar, em muitos momentos, a uma compreensão de que o desenvolvimento do processo investigativo cabe apenas à Universidade.

Aqui, partimos do entendimento oposto, ou seja, de que o processo de pesquisa não precisa (e não pode) ficar restrito ao âmbito da Universidade e nem no âmbito do pesquisador individual, principalmente, quando se trata de pesquisa operacional na área da saúde e da enfermagem. Este processo pode ser ampliado e tornarse mais criativo, mais aderente ao correto dos problemas e da realidade dos serviços de saúde quando houver a efetiva participação de profissionais que atuam nos serviços de saúde.

A cooperação conjunta Universidade e Serviços de Saúde possibilita a troca de experiências muito saudáveis para ambas as instituições. Do lado da primeira contribui com a atualização frente às rápidas mudanças que ocorrem no setor saúde e consequentemente com um ensino dinâmico, realista e atual. Do lado dos serviços proporciona uma aproximação com elementos teóricos; e para ambas, uma fluidez na relação teoria-prática e pesquisas que respondam às necessidades do processo saúde-doença, bem como à organização das práticas sanitárias.

* Professora Doutora junto ao Departamento de Enfermagem Materno-Infantil e Saúde Pública da Escola de Enfermagem de Ribeirão Preto da Universidade de São Paulo

** Professora Titular junto ao Departamento de Enfermagem Materno-Infantil e Saúde Pública da Escola de Enfermagem de Ribeirão Preto da Universidade de São Paulo

*** Enfermeiras da Secretaria Municipal da Saúde do Município de Ribeirão Preto - SP 
Pesquisar nesta relação possibilita também que não utilizemos o campo das práticas apenas como fonte empírica de dados para os trabalhos de pesquisa, mas significa aos participantes um processo de reflexão na direção da consciência política sobre saúde, favorecendo mudanças de concepções e posturas. Assim a pesquisa desta natureza pretende ir além da realização de um trabalho formal, entendido como a produção de um texto. Tem a preocupação de contribuir para que tanto profissionais dos serviços, como docentes desenvolvam uma postura crítica no cotidiano dos seus trabalhos.

Desta forma, todos têm oportunidade de participar dos vários momentos do processo de trabalho da pesquisa: a identificação e seleção dos objetos a serem pesquisados (como aqueles problemas práticos que requerem solução e/ou melhores encaminhamentos); a escolha de fundamentações teóricas e metodológicas coerentes com as transformações que se deseja operar; a divulgação dos resultados e fazer com que os mesmos sejam utilizados e/ou colocados na prática.

Agrega-se assim à ação técnico-científica a comunicativa e política. É também um processo de desmistificação da pesquisa para entendê-la como um instrumento democrático e de acesso possível àqueles que lidam diretamente com a assistência à saúde.

O Departamento de Enfermagem MaternoInfantil e Saúde Pública da Escola de Enfermagem de Ribeirão Preto-USP acompanhando o processo de Municipalização da Saúde tem encaminhado uma discussão sobre as possibilidades de transformação do atual modelo assistencial ${ }^{* * * *}$ em saúde juntamente com profissionais da Secretaria Municipal da Saúde de Ribeirão Preto - SMS-RP, que ocupam funções diretivas, gerenciais e de chefia de serviços. Este projeto conjunto denominase GRUPO DE TRABALHO E PESQUISA GERENCIAMENTO E INFORMÁTICA EM

SAÚDE e tem por finalidade articular teoria e prática, ensino e assistência e concepção e execução do trabalho em saúde coletiva.

Assim os pressupostos norteadores deste trabalho são:

- articulação interinstitucional, intergrupal e interpessoal para alcançar metas/horizontes conjuntamente construídos e definidos;

- responsabilidade compartilhada, construção conjunta, coordenação articulada do processo de encaminhamento do Grupo de Trabalho e Pesquisa Gerenciamento Local e Informática em Saúde;

- aquisição e aprofundamento de conhecimentos técnicocientíficos e uma ação comunicativa e política, a partir do exercício de reflexão acerca dos fenômenos da prática em saúde.

Este ensaio tem por objetivo relatar a experiência compartilhada do processo de pesquisar em saúde entre Universidade e Serviço de Saúde, apontando o intercâmbio desta parceira e seus resultados.

\section{DESENVOLVIMENTO DA PROPOSTA CONJUNTA}

\section{Motivação Inicial}

Em fevereiro de 1993, um grupo de docentes da Escola de Enfermagem de Ribeirão Preto da Universidade de São Paulo encaminhou à Secretaria Municipal de Saúde de Ribeirão Preto (SMS-RP), uma carta de intenções acerca da proposta de trabalho junto àquela instituição.

Neste documento foi explicitada a vocação do Departamento de Enfermagem Materno-Infantil e Saúde Pública no que diz respeito a suas áreas de conhecimento, enfatizando-se a preocupação tanto ao nível de ensino, da pesquisa e da extensão de serviços de integrar, na medida do possível, os seus conteúdos e a prática dos serviços de saúde, preocupação esta expressa na tentativa de se ter uma aproximação da teoria com a realidade prática a fim de que a Universidade possa contribuir para o encaminhamento dos problemas e anseios da sociedade.

No referido ano, com a mudança do governo municipal e a proposta de concretizar o processo de municipalização na saúde, criou-se a possibilidade de aprofundar a discussão sobre o gerenciamento das Unidades Básicas de Saúde - UBS, de forma que em julho de 1993, inicia-se o processo de indicação dos gerentes locais.

Uma vez que a Saúde Pública se constitui em uma das áreas do conhecimento do Departamento acima citado, e como grandes transformações do setor saúde vêm ocorrendo nesta última década (ALMEIDA, 1990), com o processo de municipalização, foi considerada no documento, a necessidade de continuar o trabalho de aproximação da academia com os serviços de saúde, de forma a permitir a realização de pesquisas cujos objetos de investigação emanem das necessidades da prática e os resultados também possam conduzir esta mesma prática; da mesma forma que é desejável que isto ocorra em relação ao ensino.

O Grupo iniciou no final de fevereiro de 1993,

\footnotetext{
**** Aqui tomamos o conceito de Modelo Assistencial como a forma como são produzidas as ações de saúde e a maneira como o Estado e os serviços de saúde se organizam para produzí-las e distribuí-las. (CAMPOS et al., 1989)
} 
uma série de reuniões, primeiramente com as enfermeiras de cada Distrito de Saúde ${ }^{* * * * * *}$ do município em separado, com o intuito de discutir e avaliar o interesse e a disponibilidade de aprofundamento da temática supervisão e gerência - e a possibilidade do projeto de trabalho.

O caminhar dos contatos com profissionais da SMS-RP apontou a necessidade de um trabalho que tivesse caráter multidisciplinar sendo que, já em abril de 1993, as reuniões passaram a contar com a presença de enfermeiras e de um pequeno número de médicos da SMS-RP e docentes do Departamento de Medicina Social da Faculdade de Medicina de Ribeirão Preto da Universidade de São Paulo.

\section{Objetivos do Grupo}

Quando se pensa na questão do gerenciamento dos serviços de saúde, deve-se refletir que, principalmente com a mudança da proposta de atenção à saúde através da implantação do SUS e da municipalização, muda também o enfoque do processo de gestão, uma vez que o desempenho das atividades gerenciais não pode ser visto somente como uma questão técnico administrativa, mas também política e psicossocial. (ALMEIDA et al., 1994)

Num primeiro momento, todo o trabalho do grupo que passou a se denominar GRUPO DE TRABALHO E PESQUISA GERENCIAMENTO LOCAL, foi no sentido de identificar a problemática do gerenciamento, sendo evidenciado que o mesmo, no contexto das Unidades Básicas de Saúde da SMS-RP do município de Ribeirão Preto, se voltaria para garantir a funcionalidade interna da unidade, ou seja, garantir o funcionamento em termos da infraestrutura mínima para que o atendimento ai se realizasse (alguns pontos identificados dizem respeito ao material de consumo permanente e medicação, recursos humanos, fluxo da clientela, fluxo de informação).

Em 15 de dezembro de 1993, profissionais ligados à área da informática tanto da SMS-RP como da Escola de Enfermagem, fizeram a proposta de agregar a este grupo a Informática ${ }^{* * * * *}$, por constituir-se em suporte para o gerenciamento.

Este grupo passou a denominar-se GRUPO

TRABALHO E PESQUISA GERENCIAMENTO E INFORMÁTICA EM SAÚDE, com os seguintes objetivos iniciais:

- assessorar a implantação da gerência e informática nas unidades de saúde;

- subsidiar tecnicamente as atividades de gerenciamento e informática da SMS-RP;

- contribuir para a formação e desenvolvimento de recursos humanos em gerenciamento e informática;

- implementar e contribuir para o desenvolvimento de pesquisas;

- subsidiar e colaborar no acompanhamento e avaliação das atividades de gerenciamento e informatização.

\section{As Atividades}

\subsection{Capacitação dos gerentes}

A nomeação dos gerentes se deu, entre outras razões, com a finalidade de minimizar o hiato existente entre os níveis central e distrital com nível local da SMSRP.

Os profissionais indicados para esta função apresentavam grande heterogeneidade não só quanto à formação profissional (enfermeiras, dentistas e médicos), mas também quanto ao entendimento da política atual de saúde (SUS, municipalização, etc), e principalmente quanto ao funcionamento da SMS-RP.

Mesmo entendendo ser a heterogeneidade um fator altamente estimulante para o crescimento de um grupo, percebeu-se a necessidade de, num primeiro momento, se desenvolver reflexões quanto aos temas acima citados.

Dessa forma, a realização do Curso de Gerenciamento Local $^{* * * * * * *}$, destinado prioritariamente aos gerentes de unidades, tinha por finalidade provocar a

***** Até 1994, o município de Ribeirão Preto contava com uma rede básica de saúde organizada em nível de complexidade e distribuída em 5 grandes regiões, sendo que cada região apresentava unicidade administrativa e dispunha de uma unidade de maior complexidade para referência assistencial. Estas grandes regiões, geralmente compostas por uma Unidade Básica e Distrital de Saúde e um conjunto de 3 a 9 Unidades Básicas de Saúde eram denominadas Distritos de Saúde

****** Do ponto de vista informal, já havia um intercâmbio da Informática da SMS-RP com a Escola de Enfermagem desde o final de 1992, através da participação em cursos e treinamentos na área, uso de equipamentos e softwares e assessoramento na implantação do Sistema de Notificação de Nascidos Vivos - SINASC (ROSSATO et al., 1994)

******* Os temas desenvolvidos durante o curso foram os seguintes: a construção do SUS - as políticas de saúde na última década - a municipalização dos serviços de saúde - a situação dos serviços no município de Ribeirão Preto - a Distritalização em Saúde - o relacionamento das UBS com a comunidade; o trabalho interdisciplinar em saúde; a articulação da UBS com os diferentes setores da SMS-RP - a atuação da Vigilância Epidemiológica, Vigilância Sanitária e Controle de Vetores - a administração de recursos humanos - o fluxo de informação e a informatização - a articulação da UBS com as áreas especializadas - a articulação da UBS com os serviços de apoio - as atividades e setores da UBS e sua articulação 
reflexão acerca da função do gerente local e propiciar uma instrumentalização mínima àqueles que iriam desenvolver esta atividade na SMS-RP. O conteúdo, disposto em 14 encontros, foi elaborado a partir do levantamento prévio realizado pelo Programa de Educação Contínua (PEC) da SMS-RP, junto a alguns indicados para a função, bem como dos pontos já discutidos pelo Grupo de Planejamento da SMS-RP. Para sua efetivação, contou com a participação de membros do Grupo de Trabalho e Pesquisa em coordenação conjunta com o PEC.

O curso possibilitou que os participantes tivessem uma visão mais geral acerca do funcionamento da SMSRP dentro do contexto sanitário e político atual, sendo que durante o seu desenvolvimento foi produzido um documento pelos participantes apontando estrangulamentos no trabalho gerencial e alternativas para a solução de problemas considerados emergenciais pelos gerentes. Este documento foi encaminhado ao Secretário da Saúde em janeiro de 1994.

Outro desdobramento deste processo de reflexão culminou com a montagem de um Grupo de Estudos com reuniões mensais e participação dos gerentes, chefias e Grupo de Trabalho e Pesquisa.

Este grupo tinha por finalidade garantir um espaço de estudo e discussão de problemas vivenciados no cotidiano do trabalho dos gerentes, sendo que as temáticas ${ }^{* * * * * * *}$ desenvolvidas emergiram das necessidades do próprio grupo.

Observou-se que o desenvolvimento deste Grupo de Estudos também se constitui em uma forma de fazer pesquisa, sendo esta uma pesquisa prática do cotidiano, uma vez que o referencial teórico que movia o grupo era a relação teoria-prática.

Em junho de 1994 foi realizado o módulo Chefia e Liderança, coordenado pelo PEC e que teve por objetivo proporcionar aos gerentes e chefias administrativas e integrantes do Grupo de Trabalho e Pesquisa, uma vivência em trabalho grupal, quando foram repensadas as formas de liderança: autocrática, democrática e centrada no grupo.

Em função das discussões surgidas durante os Grupos de Estudo, somadas às emergentes no módulo de Chefia e Liderança, chegou-se à necessidade de se envolver o nível central nos espaços de capacitação dos gerentes uma vez que esta não pode ocorrer desarticulada às diretrizes de trabalho da SMS-RP.

A partir desta constatação, discussões foram desenvolvidas entre integrantes do Grupo de Trabalho e Pesquisas e equipe dirigente da SMS-RP (Secretário de Saúde e Assistentes), o que culminou com a proposta do desenvolvimento de Seminários.

Paralelamente a estas ações, a Escola de Enfermagem realizou um curso de Especialização em Saúde Coletiva possibilitando a participação de profissionais da SMS-RP.

Em agosto de 1994, foi realizado seminário com a participação de um assessor da OPAS, que trouxe a discussão sobre $O$ Distrito Sanitário enquanto processo social de mudança das práticas sanitárias. Este encontro mobilizou os técnicos da SMS-RP, uma vez que foram colocados em discussão os conceitos norteadores da Reforma Administrativa que vinha sendo proposta a fim de repensar o Distrito Sanitário, numa perspectiva de mudanças das práticas sanitárias.

Em outubro de 1994, com a mudança do Secretário da Saúde do município, houve necessidade de uma readequação nas ações de educação continuada destinadas aos gerentes, em decorrência da mudança na estrutura administrativa da SMS-RP, quando os distritos administrativos foram extintos com perspectiva de aproximação entre os níveis central e local. Com isto, o Grupo de Trabalho e Pesquisa Gerenciamento e Informática em Saúde percebeu a necessidade de readequar suas atividades.

Em abril de 1995 foi organizado um encontro envolvendo lideranças do nível central da SMS-RP, em articulação do Grupo de Trabalho e Pesquisa Gerenciamento e Informática em Saúde com a PósGraduação da Escola de Enfermagem de Ribeirão PretoUSP, para discutir o processo de Territorialização em Saúde. Esta Oficina de Trabalho trouxe aos profissionais da SMS-RP a perspectiva da implantação efetiva do Distrito Sanitário enquanto processo social. Este trabalho foi coordenado pela enfermeira Maria Cristina Melo da Universidade Federal da Bahia e assessora da Secretaria Municipal da Saúde de Salvador.

Com relação à capacitação dos gerentes, o Grupo

******** As temáticas contemplaram questões como:

- constituição da gerência voltada para as questões mais internas da unidade, e para sua funcionalidade;

- definição da função do gerente, objetivando a definição de metas na unidade básica;

- falta de integração da equipe e a compreensão sobre o trabalho em equipe;

- falta/dificuldade de comunicação entre a equipe local e entre esta e os demais níveis (Distrital e Central);

- questionamento dos limites da função gerencial e das competências específicas das demais categorias profissionais;

- falta de envolvimento e compromisso por parte dos servidores públicos;

- falta de poder de decisão nos vários níveis da Secretaria;

- falta de valorização das aptidões individuais, de planejamento das ações e dos resultados;

- deficiência de recursos materiais em geral. 
de Trabalho e Pesquisa está discutindo a possibilidade da realização de Oficinas de Trabalho nas Unidades de Saúde, visando o reconhecimento do território e adequação do serviço às necessidades locais.

\subsection{O desenvolvimento de pesquisas}

Além das atividades já descritas o Grupo de Trabalho e Pesquisa tem trabalhado no desenvolvimento de investigações e na produção de pequenos ensaios que buscam refletir sobre a questão do gerenciamento e informática. Esta produção tem sido tanto coletiva como também individual, principalmente quando se trata trabalhos de teses.

Em linhas gerais, esta produção tem emergido no desenvolvimento do trabalho grupal.

\section{OS LIMITES E POSSIBILIDADES DO PROCESSO}

O trabalho conjunto Universidade e Serviço é um processo que está sempre em construção, com avanços e retrocessos, pois trata-se de uma relação social com determinações de várias ordens, desde as subjetivas e pessoais até as políticas e sociais. Esta parceria mesmo sendo de pequena dimensão, passa por estas determinações apresentando resultados e dificuldades.

O primeiro resultado que queremos apontar foi a constituição do grupo interinstitucional. No início, enfrentamos algumas dificuldades relacionadas a entender e aceitar o trabalho compartilhado entre os membros de instituições que têm finalidades diferentes.

A universidade se volta mais para a produção do conhecimento - o saber e os serviços estão mais voltados para a produção da assistência em saúde - o fazer. Analisando o processo de construção do grupo podemos dizer que a categoria poder esteve presente nas relações de trabalho e em alguns momentos estas foram conflitantes e possibilitaram aos integrantes refletir e discutir sobre suas posturas individuais, muitas vezes carregadas de preconceitos e (pre)juízos em relação ao significado e presença de uma ou outra instituição na parceria e medo de perda de espaço e poder.

Esses conflitos possibilitaram ao grupo maior compreensão do próprio trabalho e sua finalidade, o que reverteu em união, coesão, disponibilidade, identidade do grupo e construção de responsabilidade compartilhada.

Como a proposta tem por objetivo trabalhar com os gerentes das UBSs (Unidades Básicas de Saúde) visando um outro modelo assistencial em saúde na perspectiva da integração sanitária, era necessário que a equipe de nível central, que detém o poder de traçar a política de saúde do município, compartilhasse dos objetivos, promovendo o redirecionamento das práticas de saúde, dentro do possível.

Neste sentido, o Grupo procurou sempre uma aproximação com o nível decisório, bem como, estar articulado com as equipes do nível intermediário e local. Os avanços e dificuldades encontrados nesse processo foram de várias naturezas, tais como:

- concepção sobre organização das práticas de saúde, estando muito presente a concepção voltada para a assistência médica individual e instrumentos de gerência burocrático-administrativos para garantir somente a funcionalidade interna das unidades;

- como a visão da função gerencial se volta em grande parte para resolver questões de natureza técnicoadministrativa, a grande expectativa dos gerentes em relação ao Grupo de Trabalho era que o mesmo apresentasse um modelo formal técnico-normativo que pudesse ser aplicado em todas as situações internas do trabalho. No processo de estudo, reflexão e capacitação, esta visão foi parcialmente sendo modificada e ampliada para o conceito de Distrito Sanitário como processo social de mudança das práticas sanitárias (MENDES, 1993). Este constitui-se no grande desafio a ser alcançado por todo o grupo, pois requer profundas mudanças, desde as pessoais até as sociais;

- o encaminhamento das questões do trabalho em saúde esbarrou muitas vezes em aspectos relativos ao corporativismo dos diversos profissionais, bem como à distribuição e ocupação de cargos em nível central, distrital e local.

Vale a pena ressaltar que o Grupo vem ampliando a sua concepção de saúde e de organização de serviços com a adesão de outras instâncias a nível central e local, aumentando numericamente seus participantes.

Este aumento numérico é relativo aos profissionais de serviço, como também de alunos de graduação e pós-graduação engajados em projetos de pesquisa nessa área de conhecimento.

A heterogeneidade de profissionais envolvidos no Grupo de Trabalho e Pesquisa, bem como no gerenciamento das UBSs tem ampliado o âmbito das discussões e das visões no encaminhamento das questões de saúde, não se constituindo em uma barreira, pelo contrário, vem contribuindo para a consolidação do Grupo.

Sem dúvida, esta experiência de construção de um trabalho grupal vem demonstrando que os pressupostos teóricos traçados: articulação interinstitucional, responsabilidade compartilhada e a compreensão da lógica da integração sanitária na organização dos serviços de saúde, vem sendo possíveis de realização e estão sendo construídas compartilhadamente.

Trata-se de um processo que além de técnico- 
científico assenta-se na ação comunicativa e política, assim sendo após algumas metas alcançadas surgem outras, bem como os avanços são intercortados por retrocessos.

\section{THE RELATIONSHIP BETWEEN THE UNIVERSITY AND THE HEALTH SERVICES - BUILDING WORK POSSIBILITIES}

The present study aims at reporting the experience of construction of an interinstitutional relationship: University and Health Services, pointing out the possibilities and difficulties of this process.

KEY WORDS: research, research on health

\section{LA RELACIÓN UNIVERSIDAD Y SERVICIOS DE SALUD - CONSTRUCCIÓN DE POSIBILIDADES DEL TRABAJO}

Este ensayo visa relatar la experiencia de la construcción de una relación interinstitucional Universidad y Servicios de Salud, apuntando las posibilidades y dificultades de este caminar.

TÉRMINOS CLAVES: investigación, investigación en salud

\section{REFERÊNCIAS BIBLIOGRÁFICAS}

01. ALMEIDA, M.C.P. O trabalho de enfermagem e sua articulação com o processo de trabalho em saúde coletiva - rede básica de saúde do município de Ribeirão Preto. Ribeirão Preto, 1990. 297 p. Tese (Livre Docência) - Escola de Enfermagem de Ribeirão Preto, Universidade de São Paulo.

02. ALMEIDA, M.C.P.; FORTUNA, C.M.; PEREIRA, M.J.B.; MISHIMA, S.M.; VILLA, T.C.S. Gerência na rede básica de serviços de saúde de Ribeirão Preto - um processo em construção de trabalho. Rev.Bras.Enfermagem, v. 47, p. 278286, 1994.

03. CAMPOS, G.W.S.; MERHY, E.E.; NUNES, E.D. Planejamento sem normas. São Paulo: HUCITEC, 1989.
04. MISHIMA, S.M. Constituição do gerenciamento local na rede básica de saúde de Ribeirão Preto. Ribeirão Preto, 1995. 355 p. Tese (Doutorado) - Escola de Enfermagem de Ribeirão Preto, Universidade de São Paulo.

05. ROSSATO, M.A.; SCOCHI, C.G.S.; PEREIRA, E.B.; PRATALLI, M.T.R.; PEIXOTO, M.R. Implantação do SINASC no município de Ribeirão Preto. In: CONGRESSO BRASILEIRO DE INFORMÁTICA EM SAÚDE, 4, Porto Alegre, 1994. Anais, Porto Alegre, 1994. p. 178- 9, v. 1.

06. MENDES, E.V. (org). Distrito sanitário: o processo social de mudança das práticas sanitárias do Sistema Único de Saúde. São Paulo: HUCITECABRASCO, 1993. 